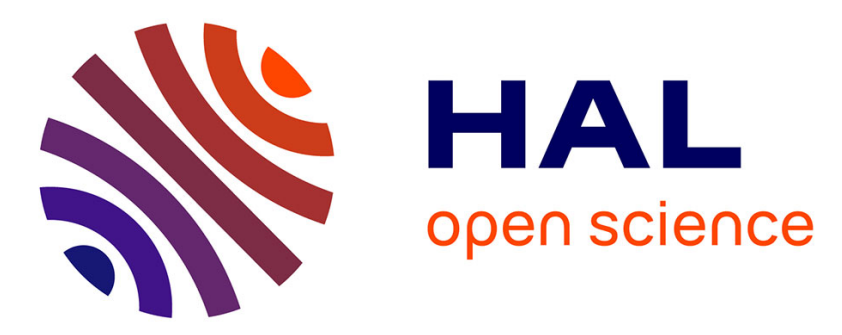

\title{
Single carrier charge collection in semiconductor nuclear detectors
}

H.L. Malm, D. Litchinsky, C. Canali

\section{To cite this version:}

H.L. Malm, D. Litchinsky, C. Canali. Single carrier charge collection in semiconductor nuclear detectors. Revue de Physique Appliquée, 1977, 12 (2), pp.303-310. 10.1051/rphysap:01977001202030300 . jpa-00244163

\section{HAL Id: jpa-00244163 https://hal.science/jpa-00244163}

Submitted on 1 Jan 1977

HAL is a multi-disciplinary open access archive for the deposit and dissemination of scientific research documents, whether they are published or not. The documents may come from teaching and research institutions in France or abroad, or from public or private research centers.
L'archive ouverte pluridisciplinaire HAL, est destinée au dépôt et à la diffusion de documents scientifiques de niveau recherche, publiés ou non, émanant des établissements d'enseignement et de recherche français ou étrangers, des laboratoires publics ou privés. 


\title{
SINGLE CARRIER CHARGE COLLECTION IN SEMICONDUCTOR NUCLEAR DETECTORS (*)
}

\author{
H. L. MALM $(* *)$, D. LITCHINSKY $(* *)$ and C. CANALI $(* * *)$
}

\begin{abstract}
Résumé. - Les détecteurs nucléaires de structure planaire nécessitent une bonne efficacité de collection des deux types de porteurs dès qu'on cherche à atteindre de bonnes résolutions en énergie. Par contre, pour des contacts sphériques, des résolutions de $3 \%$ peuvent être obtenues avec une efficacité de collection presque complète, lorsqu'un seul type de porteur est collecté. Ceci ouvre des perspectives nouvelles vers l'emploi d'autres matériaux semi-conducteurs en spectrométrie nucléaire. Les résultats théoriques et expérimentaux présentés ici indiquent une amélioration des performances en passant de structures planaires à des contacts semi-cylindriques puis hémisphériques. Ceci résulte de ce que la charge induite dépend à la fois de la géométrie, du déplacement des charges et que le piégeage diminue dans les zones où règnent des champs électriques intenses. On présente ici, pour des structures hémisphériques, les résultats d'une étude portant sur le rôle du diamètre des contacts, des produits mobilité durée de vie des deux types de porteurs, de la tension appliquée et de sa polarité sur l'efficacité de collection de photons $\gamma$. Des exemples de spectres enregistrés avec des compteurs $\mathrm{CdTe}$ et $\mathrm{HgI}_{2}$ munis de contacts hémisphériques sont présentés.
\end{abstract}

\begin{abstract}
For good energy resolution, the planar contact configuration requires good collection efficiency of both carriers. With shperical contacts, nuclear spectroscopy with energy resolutions as good as $3 \%$ and nearly full charge collection is possible with only single carrier collection. This widens the class of semi-conductors that can be used for nuclear detectors. Theoretical and experimental results demonstrate that the differences in performance obtained with planar, semi-cylindrical and hemispherical devices occur because the induced charge depends on the geometry and the charge displacement and because the charge trapping is reduced by high electric fields. For hemispherical detectors, the effects of contact radii, electron and hole mobilitylifetime products, and voltage magnitude and polarity on the values of energy resolutions and full energy efficiency for $\gamma$-rays, are shown. Examples of gamma spectra observed with hemispherical $\mathrm{HgI}_{2}$ and $\mathrm{CdTe}$ detectors are given.
\end{abstract}

1. Introduction. - By departing from the traditional plane parallel contact configuration for semiconductor nuclear detectors one can, under the proper conditions, achieve surprisingly good energy resolutions with mainly single carrier collection $[1,2]$. This feature widens the class of materials that can be used for $\gamma$-ray spectrometers. While the advantages of cylindrical and spherical configurations have long been realized in ion chambers and other types of gas tubes where the electron mobility greatly exceeds the positive ion mobility, the electric field dependence of the charge trapping and the much greater stopping power of photo (or Compton) electrons in semiconductors establish different criteria for their optimum performance as nuclear detectors.

In a planar detector with single carrier collection the spectrum is either rectangular with a long carrier

$\left(^{*}\right)$ A portion of this work was done at the California Institute of Technology. This work was supported in part by the United States Atomic Energy Commission, Atomic Energy of Canada Ltd., and the Defense Research Board of Canada, Project E-226.

(**) Aptec Engineering Ltd., Downsview, Ontario, Canada. $(* * *)$ Instituto di Fisica, Universita di Modena, Italy. drift length (relative to the contact separation), or peaked at a small fraction of full collection for a short carrier drift length [3]. For the spherical contact configuration in which the least trapped carrier is collected towards the central contact, three effects contribute to the improved performance compared to the planar contact configuration.

a) The induced charge is produced mainly in the small high field region near the small contact.

b) For most of the volume, the induced charge is nearly independent of position.

c) The trapping lifetime is proportional to the electric field.

Calculations and experimental results demonstrate that a correct choice of device parameters must be made for optimum performance. The calculations presented here are based on a simplified model for charge collection in a semiconductor detector. Similar results from a more complicated model based on Monte Carlo computations have been presented by Swierkowski [2]. The simple model gives an adequate understanding of performance expected from hemispherical devices.

With single carrier collection in a spherical configu- 
ration, a full energy peak can be achieved over a wide range of material parameters, but the variation of charge collection efficiency and detection efficiency with applied voltage is considerably different from the planar configuration. To obtain the best performance, the correct combination of inner contact radius, outer contact radius and operating voltage must be chosen according to the mobility-lifetime product of the best carrier. These features are well illustrated in this paper by the performance of $\mathrm{CdTe}$ and $\mathrm{HgI}_{2}$ hemispherical devices as $\gamma$-ray detectors.

Qualitatively, the cylindrical, elliptical and quasihemispherical configurations represent intermediate cases between the planar and hemispherical configurations. While both theoretical and experimental results obtained with cylindrical devices confirm this, these results have not been included here. However, some results are shown for quasi-hemispherical (rectangular cross-section) devices with mainly electron collection, where one contact covers five sides and the small contact is at the center of the sixth side.

While the zero space charge case (high resistivity) is discussed most extensively, the effects of a semiconductor space charge are considered qualitatively. Topics such as $\gamma$-efficiency, pulse risetimes and spatial variations are also covered.

2. Theory. - 2.1 InduCED CURRENT AND CHARge. - An understanding of nuclear detectors, in particular non-planar devices, is based on the spatial and temporal dependence of the induced current and charge. Considerable confusion has resulted however, by the misapplication of an early derivation by Ramo [4] for the induced charge and current. In a detector, the free electrons and holes produced by the stopping of a nuclear particle (e. g., photo electron, alpha-particle, etc.), are swept towards the positive and negative electrodes inducing a charge on the electrodes. A charge sensitive preamplifier is used to convert the magnitude of the charge to a voltage pulse. With a space charge, the incremental charge $\mathrm{d} q$ induced on electrode $j$ at voltage $V_{j}$ by the motion of a charge $q$ in an electric field $F$ is given by [5]

$$
\mathrm{d} q_{j}=-q\left(\frac{\partial F}{\partial V_{j}}\right)_{V} \cdot \mathrm{d} x_{j} .
$$

The induced current is

$$
i_{j}=-q\left(\frac{\partial F}{\partial V_{j}}\right)_{V} \cdot v_{j}
$$

where $v_{j}$ is the carrier velocity toward electrode $j$. For a two electrode system, the vector notation and scaler product may be dropped. This differential form as derived by Gunn [5] is equivalent to an earlier integral form presented by Beck [6]. Only in a space charge free medium is the earlier form, $\mathrm{d} q=q(F / V) \mathrm{d} x$, given by Ramo [4], valid.
From expression (1) for the planar configuration with a fixed space charge

$$
\mathrm{d} q=\frac{q \mathrm{~d} x}{x_{\mathrm{D}}}
$$

where $x_{\mathrm{D}}$ is the depletion width, while for a medium with zero space charge

$$
\mathrm{d} q=\frac{q \mathrm{~d} x}{w}
$$

where $w$ is the electrode spacing.

In the cylindrical configuration the equivalent expressions are

$$
\mathrm{d} q=\frac{q \mathrm{~d} r}{r \ln \left(r_{\mathrm{D}} / r_{1}\right)}
$$

and

$$
\mathrm{d} q=\frac{q \mathrm{~d} r}{r \ln \left(r_{2} / r_{1}\right)}
$$

for space charge and zero space charge respectively. The inner and outer radii are $r_{1}$ and $r_{2}$ respectively while $r_{D}$ is the radius of depletion, assuming depletion from the central contact. In the spherical configurations

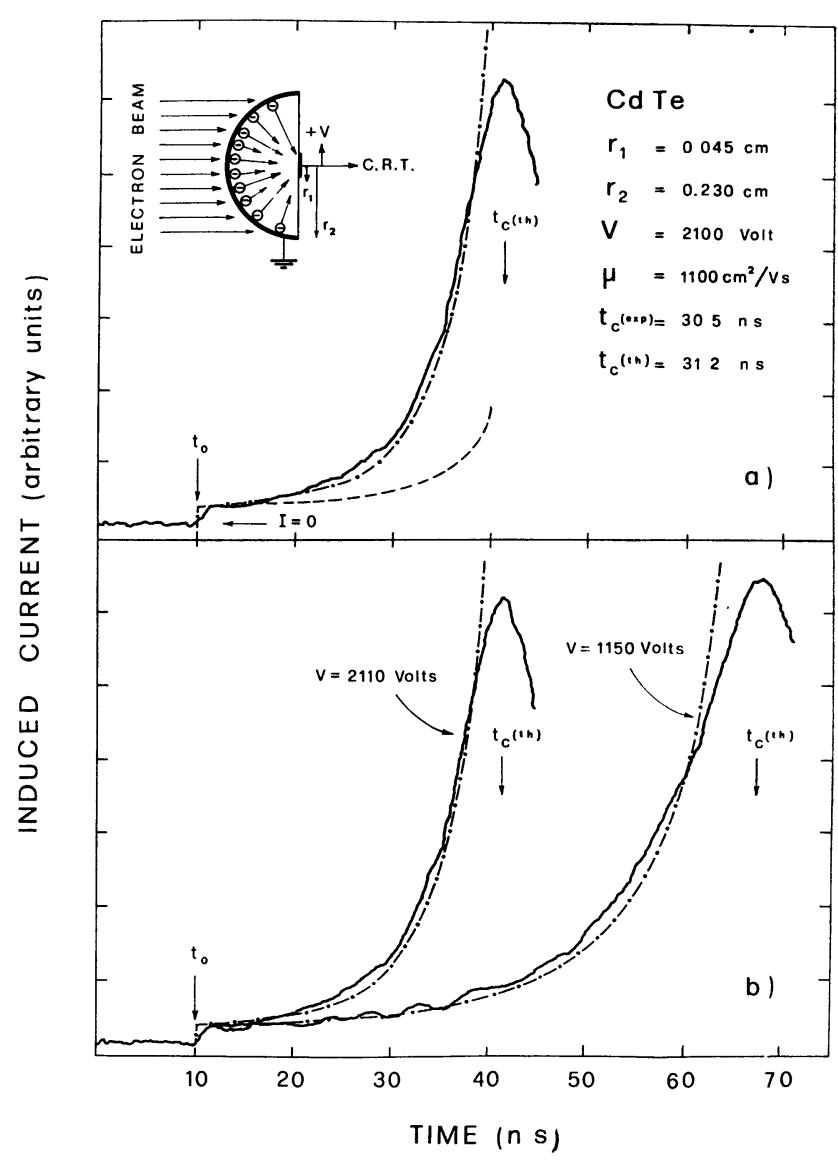

FIG. 1. - The current induced in a hemispherical CdTe crystal from a burst of electrons incident on the outer contact is shown. The dash-dot line gives the theoretical values. 
the induced charge with a fixed space charge and depletion from $r_{1}$ is

$$
\mathrm{d} q=\frac{q \mathrm{~d} r}{r^{2}\left(\frac{1}{r_{\mathrm{D}}}-\frac{1}{r_{1}}\right)}
$$

and for zero space charge $r_{\mathrm{D}}$ is replaced by $r_{2}$. Note that $\mathrm{d} q$ is only a function of geometry and position and not of electric field profile, even with a non-zero space charge.

However, the induced current dœs depend on the electric field because of the dependence of carrier drift velocity, $v_{\mathrm{d}}$, on electric field. Here we assume $v_{\mathrm{d}}=\mu F$ where $\mu$ is the carrier mobility.

For the space charge free spherical case, the induced current is given by

$i(t)=\frac{\mathrm{d} q}{\mathrm{~d} t}=\frac{q \mu V}{\left(\frac{1}{r_{1}}-\frac{1}{r_{2}}\right)^{2}}\left[r_{0}^{3}-\frac{3 \mu V t}{\left(\frac{1}{r_{1}}-\frac{1}{r_{2}}\right)}\right]^{-4 / 3}$

for charge produced at $r_{0}$. The validity of this expression was checked by an experiment where a pulsed electron gun was used to generate the charge at $r_{0}=r_{2}$ [7]. In figure 1 , the observed induced current is compared to that calculated from expression (8), (dot-dashed line). The dashed line shows the result obtained by including an incorrect geometrical factor (i. e., by using expression (3) rather than (8) to calculate the induced current). The agreement between experiment and expression (8) is good except where $t \rightarrow t_{\mathrm{c}}$, the total charge collection time. The bandwidth of the electronics and the reduced mobility at high fields distort the observed results when $t \rightarrow t_{\mathrm{c}}$. Note that in these experiments the measured charge collection time is correctly given by

$$
t_{\mathrm{c}}=\frac{\left(\frac{1}{r_{1}}-\frac{1}{r_{2}}\right)\left(r_{2}^{3}-r_{1}^{3}\right)}{3 \mu V}
$$

The agreement between theory and experiment confirms that the induced charge and electric field used for the induced current expression are correct.

With both the cylindrical and spherical configurations, most of the charge and hence most of the signal is produced by carrier travel near the inner contact. Trapping is described by introducing a mean free carrier drift times $\tau_{\mathrm{e}}^{+}$and $\tau_{\mathrm{h}}^{+}$, where the number of carriers $N$ remaining from $N_{0}$ created at time $t=0$ is given by

$$
N=N_{0} \exp \left(-\frac{t}{\tau^{+}}\right) \text {. }
$$

For the planar configuration with electron collection the total induced charge $Q_{\mathrm{e}}$ is

$$
Q_{\mathrm{e}}=\int_{0}^{x} N_{0} \mathrm{~d} q=N_{0} q \frac{(\mu \tau)_{\mathrm{e}} F}{w}\left[1-\exp \left(-\frac{x}{(\mu \tau)_{\mathrm{e}} F}\right)\right] .
$$

A similar expression follows for hole collection. This form was used by Akutagawa [3] to give single carrier spectra from planar devices.

In the s pherical configuration for electron collection towards the central positive contact

$$
Q_{\mathrm{e}}=\frac{N_{0} q r_{1} r_{2}}{\left(r_{2}-r_{1}\right)} \int_{r_{0}}^{r_{1}} \exp \left[\frac{\left(r^{3}-r_{0}^{3}\right)\left(r_{2}-r_{1}\right)}{3(\mu \tau)_{\mathrm{e}} V r_{1} r_{2}}\right] \frac{\mathrm{d} r}{r^{2}}
$$

where $r_{1}$ and $r_{2}$ are the inner and outer radii respectively. For hole collection towards the outer contact

$$
Q_{\mathrm{h}}=\frac{N_{0} q r_{1} r_{2}}{r_{2}-r_{1}} \int_{r_{0}}^{r_{2}} \exp \left[\frac{\left(r_{0}^{3}-r^{3}\right)\left(r_{2}-r_{1}\right)}{3(\mu \tau)_{\mathrm{h}} V r_{2} r_{1}}\right] \frac{\mathrm{d} r}{r^{2}} \text {. }
$$

For these calculations the values of $(\mu \tau)_{e}$ and $(\mu \tau)_{h}$ are assumed to be field independent ; this is a good approximation but some departures from this may be observed in practice $[8,9]$.

Similar expressions can be written for cylindrical electrodes.

2.2 Pulse Height Spectra. - A pulse height spectrum is a plot of $\mathrm{d} n / \mathrm{d} Q$ vs $Q$ where $\mathrm{d} n$, the number of events in the induced charge interval, is proportional to the number of fully absorbed $\gamma$-rays per unit volume, and $Q$ is linearly proportional to the nuclear particle energy. For no charge trapping a simple analytic form exists for spherical contacts [1] ; the pulse height distributions for mainly single carrier collection with trapping, shown in figure 2, were obtained with the use of a computer. Only full energy events were considered ; Compton and electron escape effects are not included. While a small hole $\mu \tau$ value was included, the effect of hole collection is almost negligible until the hole $\mu \tau$ value is greater than 0.05 of that for the electrons. As well, a uniform ionization density was assumed. This condition is most closely approached for $\gamma$-ray energies $>200 \mathrm{keV}$.

The most salient feature of the hemispherical device is that a peak in the $\gamma$-ray spectrum occurs only when the better (lesser trapped), carrier is collected towards the center contact. A second feature is that the full 


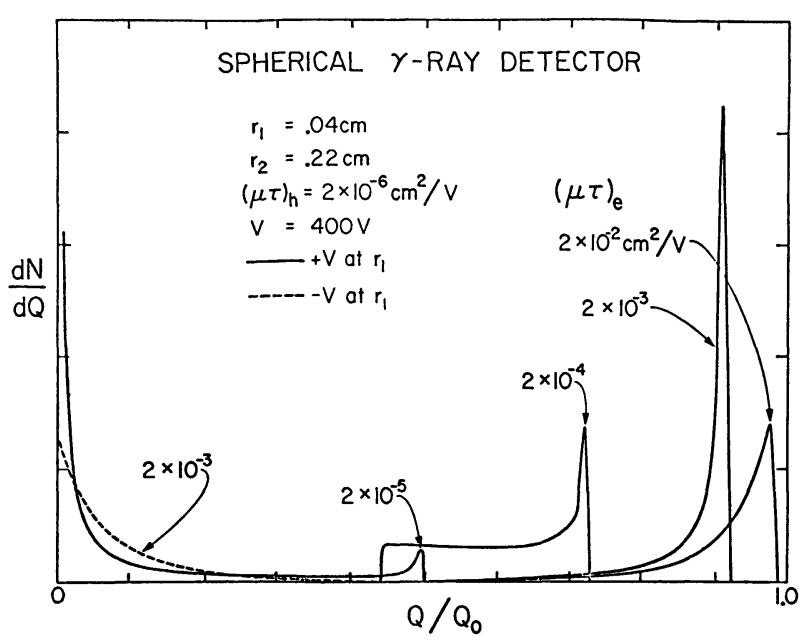

Fig. 2. - Calculated pulse height distributions for the full energy peak for a spherical device. The spectra are shown for $(\mu \tau)_{\mathrm{e}}$ values of $2 \times 10^{-5}$ to $2 \times 10^{-2} \mathrm{~cm}^{2} / \mathrm{V}$ and $(\mu \tau)_{\mathrm{h}}$ of $2 \times 10^{-6} \mathrm{~cm}^{2} / \mathrm{V}$. The solid lines apply when the inner electrode is positive and the dashed line applied when this electrode is negative.

energy peak resolution is best at less than full collection in contrast with two carrier collection in planar devices. A third feature of the single carrier spectra is that a peak results even when the average electron drift length is much less than the electrode spacing. This peak occurs because $\mathrm{d} Q / \mathrm{d} r$ can actually pass through a maximum when $(\mu \tau)_{\mathrm{e}} \gg(\mu \tau)_{\mathrm{h}}$ in nonplanar configurations. Even though a peak is observed, the full energy peak efficiency may be much less than that expected from the total detector volume. This feature is shown in figure 3 , where the efficiency is defined by that percentage of the pulses which have amplitudes between 80 and $100 \%$ of the observed peak. Note that the charge collection efficiency approaches $100 \%$ very slowly with $\mu \tau V$ while the $\gamma$-efficiency rises steeply and, for the above definition, gees through a

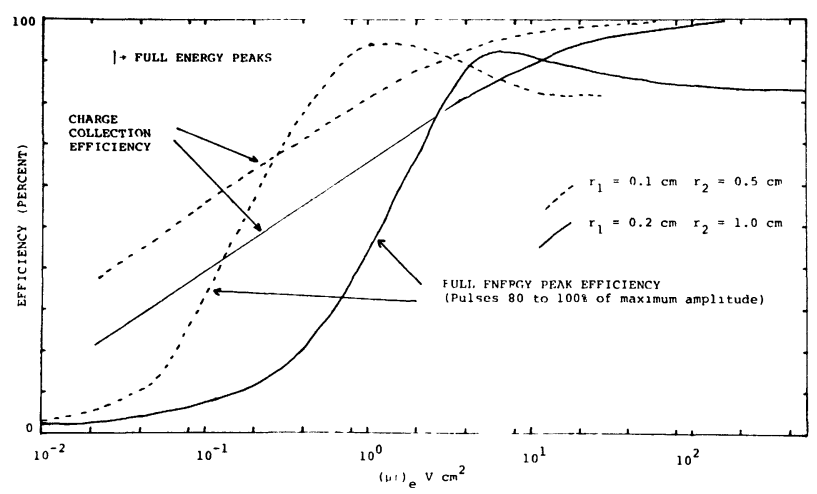

FIG. 3. - The charge collection efficiencies and the detection efficiencies are shown as functions of the $\mu \tau V$ of the carrier collected to the center contact for two sets of values for $r_{1}$ and $r_{2}$. Full (100\%) charge collection efficiency was determined letting $\mu \tau V \rightarrow \infty$. The detection efficiency was defined by that fraction of the total detector volume which gave pulses in the range 80 to $100 \%$ of the amplitude for the full energy peak. maximum. For a smaller value of $r_{1} / r_{2}$ or for a larger energy window this maximum disappears.

3. Experimental results. - 3.1 ChARge colleCTION EFFICIENCY. - When $\mu \tau V$ is very large, $Q_{\mathrm{e}}(r)$ increases monatonically with distance from the center, while for small $\mu \tau V$, the value of $Q_{\mathrm{e}}(r)$ passes through a maximum value which depends on the value of $\mu \tau V$. At intermediate values of $\mu \tau V$ the induced charge is nearly constant for a considerable range of $r$; this is the condition for a narrow peak in a $\gamma$-ray spectrum.

Certain aspects of this predicted behaviour of $Q_{\mathrm{e}}$ versus $r$ were observed by performing a $60 \mathrm{keV}$ collimated $\gamma$-ray scan of a hemispherical CdTe device for which the electron $\mu \tau$ product of $1.5 \times 10^{-3} \mathrm{~cm}^{2} / \mathrm{V}$ was almost 1000 times that for holes (Fig. 4). Because

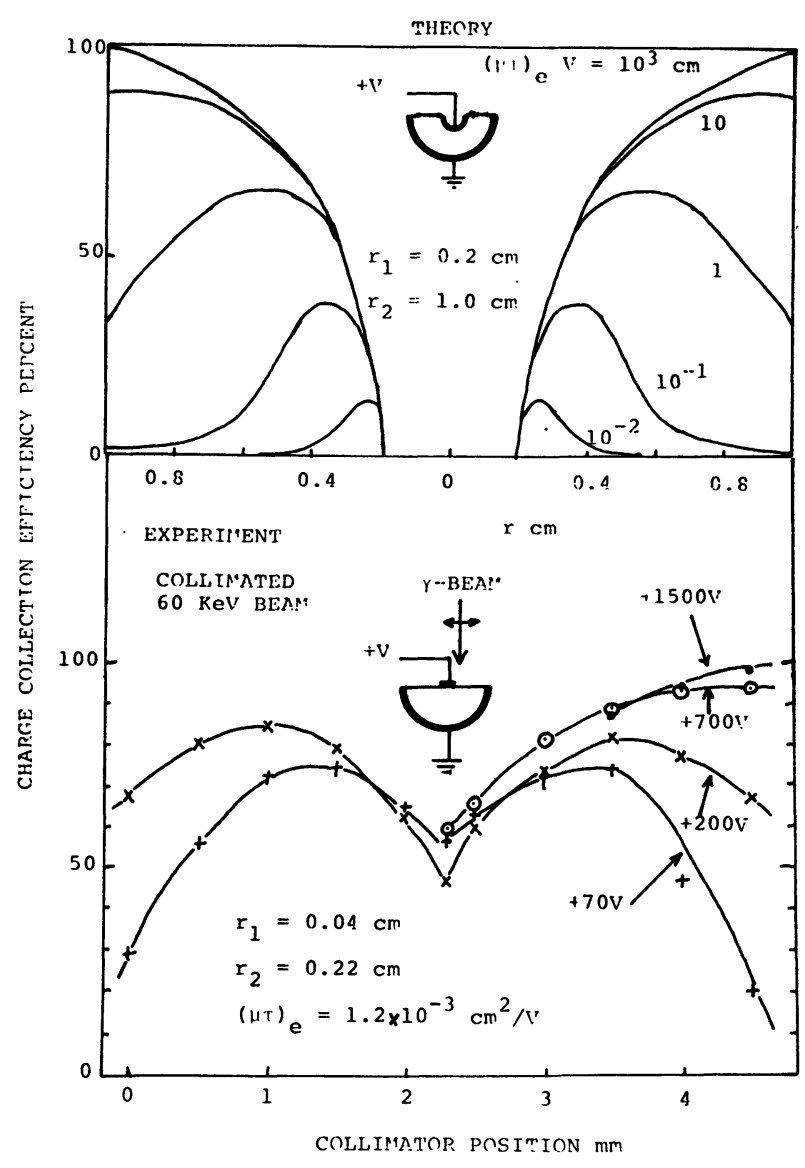

FIG. 4. - In the top portion the variation of electron charge induced as a function of radial position was calculated for several values of $(\mu \tau)_{\mathrm{e}} V$ in a hemispherical device. The $0.05 \mathrm{~mm}$ diameter collimated beam of $60 \mathrm{keV} \gamma$-rays was used to generate carriers near the surface of the hemispherical CdTe detector. The experimental positions of the ${ }^{241} \mathrm{Am}$ peak show that the variations of shape of the $Q_{\mathrm{e}}$ vs position curves with voltage are similar to that predicted by theory.

of the short absorption depth $(0.025 \mathrm{~cm})$ for this energy of $\gamma$-rays, the measurements of relative $\gamma$-ray peak amplitudes give the charge collection efficiencies along a radius.

Note that the radial profile of the charge collection 
efficiency in the experimental results changes with voltage in agreement with that shown by the theoretical calculation in the top portion of figure 4. Near the center contact the departure from spherical geometry and the neglect of hole traversal in the top part of figure 4 cause differences between theory and experiment.

3.2 Pulse height Spectra. - Both CdTe and $\mathrm{HgI}_{2}$ have been used in the hemispherical configuration. The optimization of performance depends quite strongly on the values of $r_{1}, r_{2}$ and the $(\mu \tau)_{\mathrm{e}}$ and $(\mu \tau)_{\mathrm{h}}$ products. Spectra shown in figure 5 demonstrate some of these features.
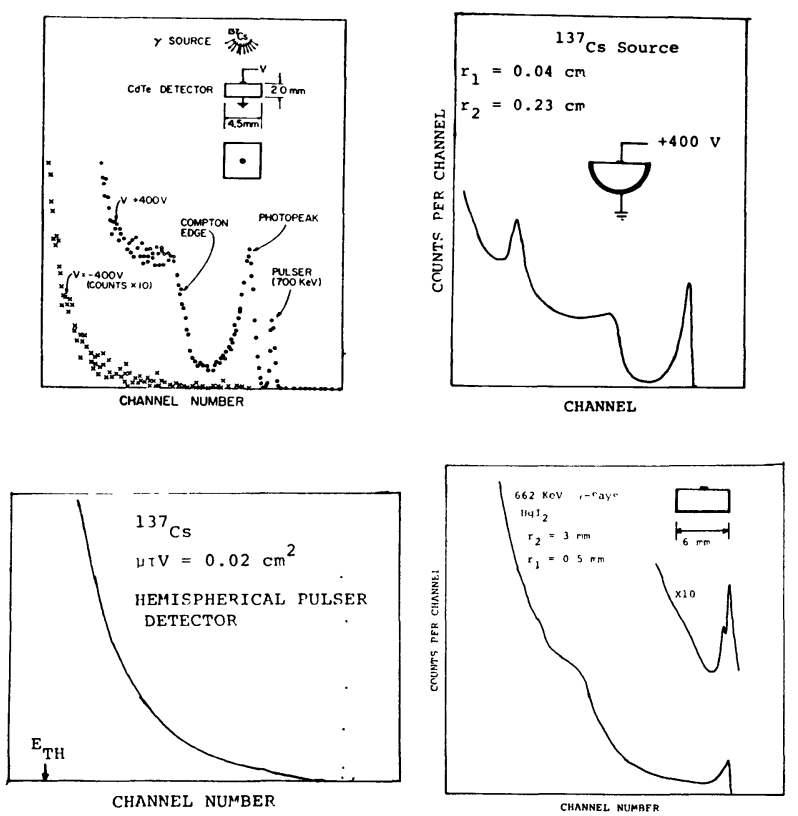

Fig. 5. - Single carrier $\gamma$-ray spectra for $\mathrm{CdTe}$ and $\mathrm{HgI}_{2}$ devices. In the top portion the effect of rounding off the corners is shown. As well, the effect of the wrong bias voltage is shown by the dramatically reduced charge collection and efficiency. In the lower portion it is shown that a CdTe device with a relatively small value of $\mu \tau V$ operates satisfactorily as a detector, while the quasi-hemispherical $\mathrm{HgI}_{2}$ device shows a peak even when the average electron drift length is less than the device size.

The spectrum of $662 \mathrm{keV} \gamma$-rays taken with a quasihemispherical CdTe detector, in the upper left corner, demonstrates the tremendous difference in performance between positive and negative voltages on the small contact. The difference is from the different sensitive volumes for electron and hole collection. With plane parallel contacts the spectrum was rectangular and no peaks could be seen.

The spectrum in the upper right corner, taken with a more nearly hemispherical device, shows that some improvement is achieved by eliminating the corner regions. The peak to valley ratio described by the full energy peak height to the valley between the Compton edge and the peak was improved from $7: 1$ for the rectangular cross-section device to $16: 1$ for the semicircular cross-section device.
In the lower left corner a ${ }^{137} \mathrm{Cs}$ spectrum is shown taken with a $2 \mathrm{~mm}$ radius device for which $\mu \tau V$ was only $2 \times 10^{-2} \mathrm{~cm}^{2}$. While the full energy peak is not visible, this device performs well as a low voltage radiation detector because the average charge collection efficiency $(\simeq 50 \%)$, is sufficient to ensure that most pulses exceed the noise threshold, $E_{\mathrm{th}}$. This is often the requirement of a medical probe. With a $2 \mathrm{~mm}$ planar device the above value of $\mu \tau V$ would give an average charge collection efficiency of only about $20 \%$.

Finally, in the lower right section of figure 5, a ${ }^{137} \mathrm{Cs}$ spectrum from a $\mathrm{HgI}_{2}$ crystal reported by Puschert [10] is shown. Even though the value of $\mu \tau V$, estimated to be 0.1 , is too small to ensure a large peak efficiency, a full energy photopeak is observed, at greater than $50 \%$ charge collection efficiency. The photopeak portion of the spectrum is similar to that shown by the curve labelled $2 \times 10^{-4}$ in figure 2 . Another hemispherical $\mathrm{HgI}_{2}$ detector spectrum reported by Whited [11] shows good ${ }^{137} \mathrm{Cs}$ resolution but only after a polarization occurs which likely contributes a space charge, the effects of which are not considered in detail here.

When the electron and hole mobility-lifetime proJucts approach each other, the hemispherical devices have poorer performance than planar devices. $\mathrm{A}{ }^{137} \mathrm{Cs}$ spectrum shown in figure 6 , taken with a $3 \mathrm{~mm}$ dia-

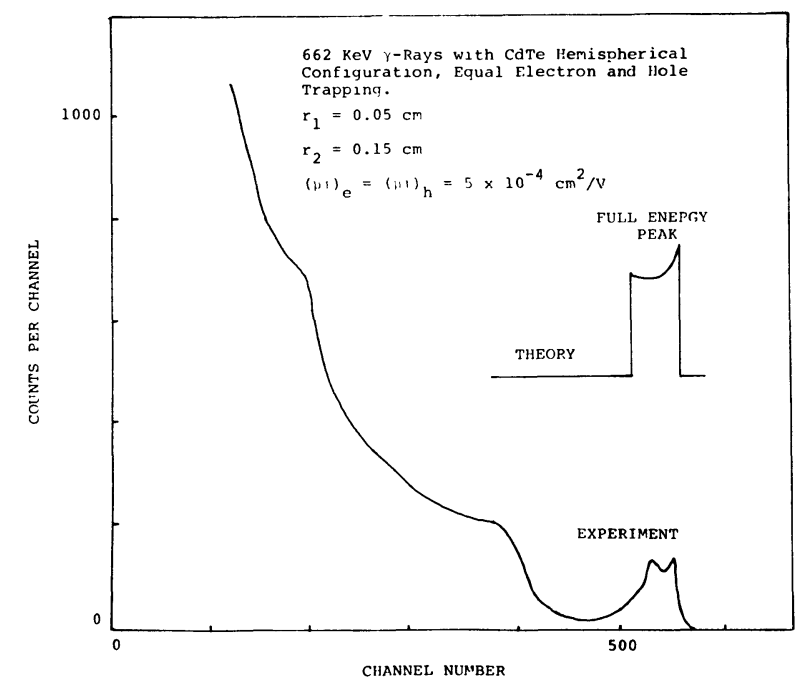

Fig. 6. - When the electron and hole $\mu \tau$ products are nearly equal, the spherical configuration degrades the performance. With a larger value of $r_{2}$ the full energy peak broadens even more.

meter device, shows this effect. Here the electron and hole $\mu \tau$ products are both estimated to be $5 \times 10^{-4} \mathrm{~cm}^{2} / \mathrm{V}$ but the peak width both from calculations and experiment is wider than that expected from a $1 \mathrm{~mm}$ planar device [12]. As a general rule, the non-planar devices show better performance when the mobility-lifetime products differ by more than a factor of about 10 . 
3.3 Resolutions. - From theory, full energy resolutions to about $3 \%$ can be expected with single carrier hemispherical devices by the optimum choice of detector parameters. Crystal inhomogeneities, difficulties in fabricating truly spherical contacts and electronic noise all contribute to poorer resolutions. As suggested by figure 2, the resolutions do exhibit an optimum with respect to $\mu \tau V$. This feature is shown in figure 7 by a plot of the resolution as a function of detector voltage. The best ${ }^{137} \mathrm{Cs}$ resolution observed with this device was $5.5 \%$.

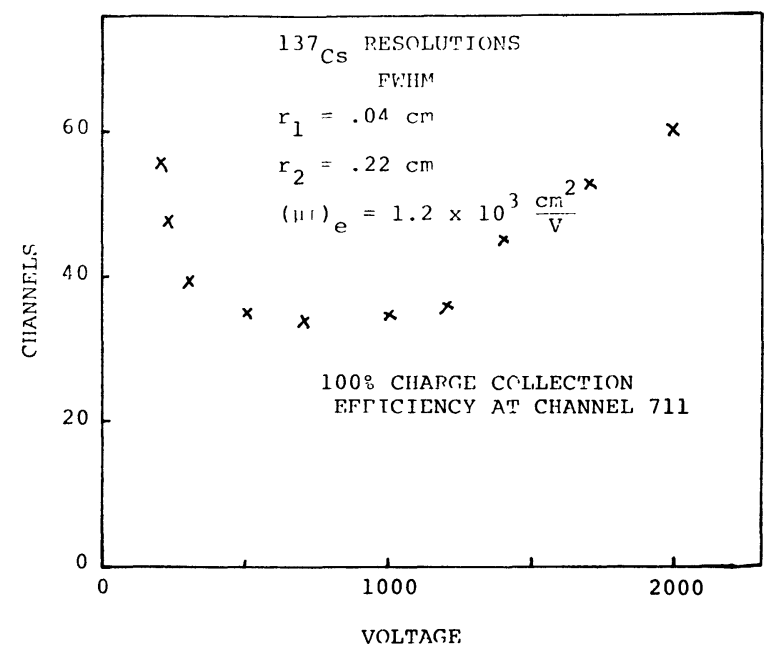

FIG. 7. - With a hemispherical single carrier device an optimum resolution is reached at an intermediate value of $\mu \tau V$. The resolutions shown here were obtained by subtracting the pulser width from the observed peak width in quadrature.

3.4 Efficiencies. - In figure 8 the charge collection efficiency of the $662 \mathrm{keV} \gamma$-ray peak for a device with an electron $\mu \tau$ product of about $1.5 \times 10^{-3} \mathrm{~cm}^{2} / \mathrm{V}$

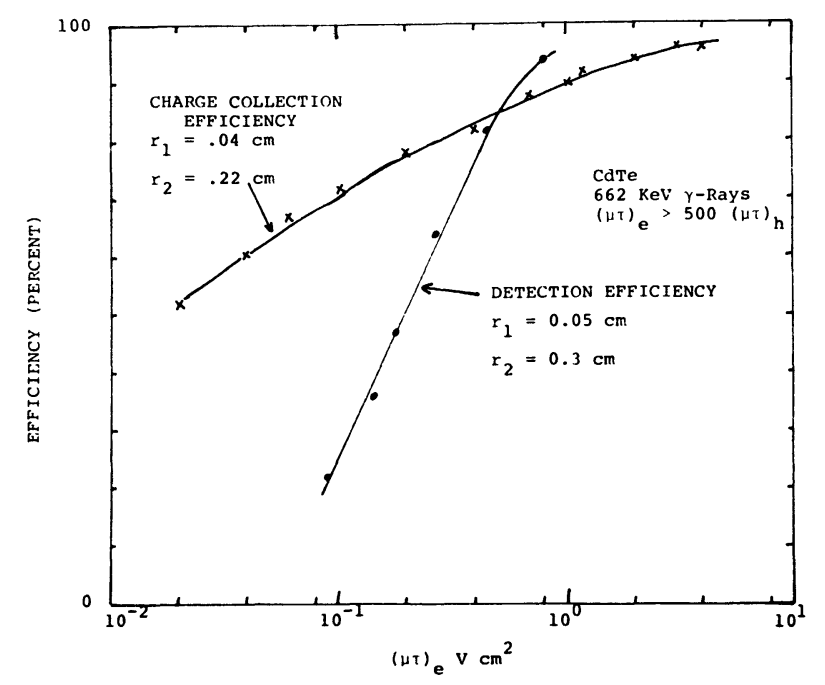

FIG. 8. - The experimental measurements of collection efficiency. Full (100\%) charge collection efficiency was obtained with a $\mathrm{Ge}(\mathrm{Li})$ diode and the values of $2.98 \mathrm{eV}$ and $4.46 \mathrm{eV}$ for the energies per electron-hole pair for Ge and CdTe respectively. The $\gamma$-efficiency for pulses of 75 to $100 \%$ of full amplitude was taken from reference [15] is plotted as a function of voltage. Full, (100\%) collection efficiency was calculated from the $\gamma$-ray pulse heights of a $\mathrm{Ge}(\mathrm{Li})$ detector and from the published energies per electron-hole pair of $2.98 \mathrm{eV}$ for Ge [13] and 4.46 for CdTe [14]. Also plotted is the detection efficiency obtained from Malm et al. [15] where a quasi-hemispherical device (5-sided negative contact), with an electron $\mu \tau$ value of $9 \times 10^{-4} \mathrm{~cm}^{2} / \mathrm{V}$ was measured. Pulses falling in the range 75 to $100 \%$ were included. The agreement with the theoretical plots of figure 3 is good. Similar results have been presented by Zanio [16].

These results demonstrate that, unlike planar devices, the detection efficiency can vary many times over a fairly small voltage range, while the charge collection efficiency, or peak height, varies much less with detector voltage.

3.5 Spatial Sensitivity. - With single carrier collection, it is expected that the region just below one of the contacts will have very poor charge collection. This feature has indeed been observed for quasi-hemispherical CdTe devices $[15,17]$ in which the value of $(\mu \tau)_{\mathrm{e}}$ was likely much greater than $(\mu \tau)_{\mathrm{e}}$. This behaviour, shown in figure 9 by scans with a collimated beam, indicates a thin dead layer just under the positive contact. When the polarity is reversed this dead layer

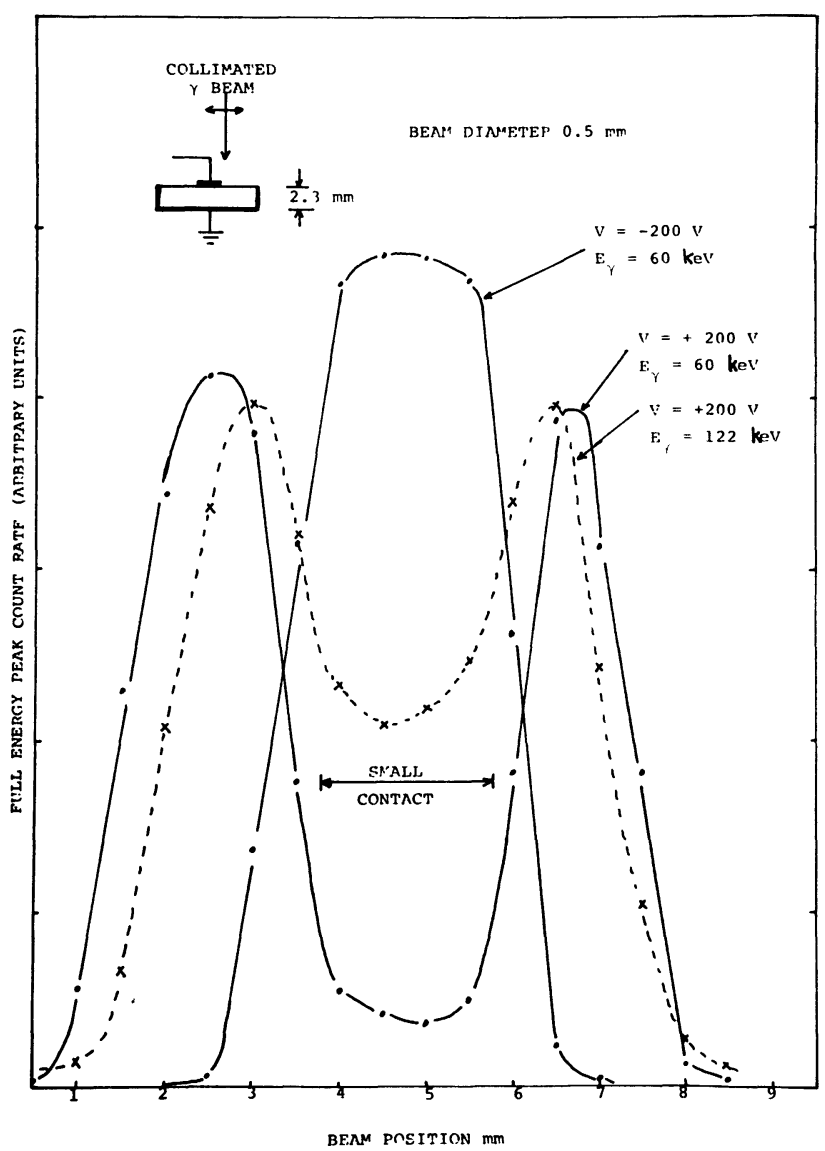

FIG. 9. - Spatial efficiency variations for low energy collimated $\gamma$-ray beams show the local effects of single carrier collection (electron) in this CdTe. 
disappears as the induced charge in this region is now generated by electron collection which is much better than the hole collection.

Because of this spatial variation in sensitivity for low energy $\gamma$-rays entering the small contact side, low energy $\gamma$-rays in a single carrier device are best observed incident on the large negative contact [18]. Since the volume of reduced sensitivity is normally very small, the effect of source orientation for weakly absorbed $\gamma$-rays like those from ${ }^{137} \mathrm{Cs}$ is small.

3.6 Pulse Risetimes. - Because most of the induced current is generated in a very short time in a hemispherical device with $r_{1} / r_{2}<0.2$, the charge pulse risetimes are fast. For example, with a $2.5 \mathrm{~mm}$ CdTe planar device, the electron and hole risetimes are, for full traversal and a $200 \mathrm{~V} / \mathrm{mm}$ field, $0.12 \mu \mathrm{s}$ and $1.2 \mu \mathrm{s}$ respectively, thus limiting the integration time constants to about $2 \mu \mathrm{s}$. With a $2.5 \mathrm{~mm}$ radius hemispherical device, the maximum risetime for 5 to $95 \%$ of full amplitude is reduced, for $r_{1} / r_{2}=0.2$ and $V=500 \mathrm{~V}$, to less than $0.1 \mu \mathrm{s}$ for all pulses. This allows the use of very short integrating time constants and allows very high counting rates. With a quasi-hemispherical CdTe device, a full energy ${ }^{137} \mathrm{Cs}$ peak has been observed even with an amplifier integrating time constant as short as 63 ns.

4. Discussion. - 4.1 Zero SPACE CHARGE DEVICES. - With hemispherical or even quasi-spherical contacts, good spectrometer performance can be achieved with single carrier collection. In the spherical devices, most of the induced charge is generated near the inner contact and the amount of charge lost by trapping is electric field dependent. The theory and results show that this implies that the contact sizes and operating voltage must be correctly chosen in relation to the $\mu \tau$ product of the best carrier to ensure that the optimum performance is achieved.

Resolutions of a few percent can and have been achieved with hemispherical $\mathrm{CdTe}$ and $\mathrm{HgI}_{2}$ devices. Some of the other features characteristic of hemispherical devices can be beneficial in many applications where resolution is not of paramount importance. Such features include good performance at very high count rates, a good size to performance ratio, and a convenient shape for mounting.

Where better resolutions are required, one must use planar devices where the drift lengths of both carriers greatly exceed the active width. The very high fields which exist near the inner contact may cause current injection and noise ; this places a lower limit on the inner contact size. Excess contact noise sometimes occurs but in many crystals is not a serious problem. It should be noted that the current in many of the devices is small because of space charge limited current [19] for electrons because of electron traps. It may be that the even shorter trapping times for holes minimizes current injection from the positive contact, again because of space charge limited current.

4.2 Finite SPACE CHARge DEvices. - While the performance of hemispherical devices with a space charge is not discussed in detail here, some general observations can be made. Since the radial dependence of the induced charge dœs not depend on the space charge, the influence of the space charge is found in the effective device radius and in the amount of charge trapping as this is field dependent. With a positive voltage on the small contact, a negative space charge (p-type material), could give a depletion radius $\left(r_{\mathrm{D}}\right)$ less than $r_{2}$ and increase the field and thereby reduce the trapping for $r \rightarrow r_{1}$, the inner contact radius. Since the amount of trapping is already small, the high field given by the negative space charge is not beneficial and is most likely to cause problems from current injection or surface breakdown. However, with a positive voltage on the small contact and a positive space charge, the depletion would start from the outer contact. Then the high field for $r \rightarrow r_{2}$ would decrease the charge trapping, while not affecting the $1 / r^{2}$ dependence of the induced charge. One might therefore, under optimum conditions, expect improved performance in a spherical device with a negative space charge in the $10^{11} / \mathrm{cm}^{3}$ range.

5. Conclusions. - In the zero space charge hemispherical nuclear detectors, the observed performance of $\mathrm{CdTe}$ and $\mathrm{HgI}_{2}$ devices agrees with that expected from a simplified theory based on the details of the carrier collection. It was shown that materials in which the mobility-lifetime product of one carrier is more than ten times greater than that for holes, will perform better with a hemispherical rather than with a planar contact configuration. The quality of spectra depends on the relative magnitudes of the mobilitylifetime voltage product and on the inner and the outer contact radius. A hemispherical CdTe device operated with these parameters near the optimum values with electron collection gave a $662 \mathrm{keV} \gamma$-ray resolution (FWHM) of $5.5 \%$ compared to the threshold limit of $3 \%$. Because of their combination of good charge collection efficiency, good detection efficiency, and convenient shape, hemispherical devices are suitable for many applications.

Acknowledgments. - The authors gratefully acknowledge the encouragement and technical and scientific assistance provided mainly by J. W. Mayer, M.-A. Nicolet, I. L. Fowler, K. R. Zanio, and G. V. Walford. 


\section{References}

[1] Malm, H. L., Canali, C., Mayer, J. W., Nicolet, M.-A., Zanio, K. R. and Akutagawa, W., Appl. Phys. Lett. 26 (1975) 344.

[2] SWIERKowski, S. P., IEEE Trans. NS-23 (1976) 131.

[3] Akutagawa, W. and Zanio, K. R., J. Appl. Phys. 40 (1969) 3838.

[4] Ramo, S., Proc. IRE 27 (1939) 584.

[5] GunN, J. B., Solid State Electron. 7 (1964) 739.

[6] BECK, A. H. W., in Thermionic Valves (Cambridge University Press) 1953, p. 189.

[7] Canali, C. and MALM, H. L., Nucl. Instrum. and Methods 134 (1976) 199.

[8] Canali, C., Martini, M., Ottaviani, G. and Zanio, K. R., Solid State Commun. 9 (1971) 163.

[9] Malm, H. L., Submitted to J. Appl. Phys.

[10] Puschert, W. and Scholz, H., Appl. Phys. Lett. 28 (1976) 357.
[11] Whited, R. C., Schieber, M. M. and Randtke, P. T., J. Appl. Phys. 47 (1976) 2230.

[12] Triboulet, R., Cornet, A., Marfaing, Y. and Siffert, P., J. Appl. Phys. 45 (1974) 2759.

[13] Pehl, R. H., Goulding, F. S., Landis, D. A. and LenzliNGER, M., Nucl. Instrum. and Methods 59 (1968) 45.

[14] Cornet, A., Siffert, P., Coche, A. and Triboulet, R., Appl. Phys. Lett. 17 (1970) 432.

[15] Malm, H. L., Raudorf, T. W., Martini, M. and Zanio, K, R., IEEE Trans. NS-20 (1973) 500.

[16] Zanio, K., Krajenbrink, F. and Montano, H., IEEE Trans. NS-21 (1974) 315.

[17] ZANIO, K., private communication.

[18] Zanio, K., Montano, H. and Krajenbrink, F., Appl. Phys. Lett. 27 (1975) 159.

[19] Canali, C., Nicolet, M.-A. and MaYer, J. W., Solid State Electron. 18 (1975) 871. 